\title{
The Effect of Plank Exercises with Hip Abduction Using Sling on Trunk Muscle Activation in Healthy Adults
}

\author{
Jongwoo Bak, Suyoung Shim, Minkwon Cho, Yijung Chung \\ Department of Physical Therapy, College of Health Science and Social Welfare, Sahmyook University, Seoul, Korea
}

Purpose: The purpose of this study is to investigate the effects of healthy persons performing a plank exercise with hip abduction and use of sling on trunk muscle activity.

Methods: Twenty-three subjects participated in this study. Muscle activities of the multifidus, rectus abdominis, external oblique and internal oblique were assessed with electromyography (EMG) while the subjects performed 6 various types of plank exercises in random order.

Results: There was a significant difference in multifidus and internal oblique on the supported side and the multifidus, external oblique, and internal oblique on the elevated side $(p<0.05)$, and differences in plank methods were significantly higher in the multifidus, rectus abdominis, external oblique, internal oblique of the supported side and the rectus abdominis, external oblique and internal oblique on the elevated side $(p<05)$. In addition, post-hoc analysis of the various plank methods showed that muscle activity was significantly higher during the general plank exercise compared with the knee-supported-in-sling plank exercises $(p<0.05)$, and was significantly higher with the ankle-supported-in-sling plank exercises compared with the general plank exercise.

Conclusion: There was greater trunk muscle activity with plank exercises performed with the ankle-supported-in-sling and hip abduction plank exercises compared with the general or knee-supported-in-sling plank exercises. Through this study, various plank exercises have been discovered that can be selected at different intensities for core muscle training purposes based on trunk muscle strength.

Keywords: Plank, Core, Muscle activation, EMG, Hip abduction, Sling

\section{INTRODUCTION}

The trunk muscles play a pivotal role in stabilizing the spine, providing free movement of the upper and lower extremities, and has an effect on achieving a stable posture, including standing posture. ${ }^{1}$ In order to perform various movements during every day life, it is important to maintain proper trunk muscle strength and endurance because the abdominal and trunk muscles are closely related to the stability of the lower trunk, and thus trunk muscles are important for trunk movement and postural control. ${ }^{2}$ Due to the rise in interest on trunk stabilization, studies on core exercise has been reported in various fields, such as physical therapy, yoga, and pilates. ${ }^{3}$

Plank exercises are not only used for rehabilitation purposes, but are also generally used to promote physical abilities. ${ }^{4}$ Plank exercises

Received May 1, 2017 Revised Jun 9, 2017

Accepted Jun 30, 2017

Corresponding author Yijung Chung

E-mail yijung36@syu.ac.kr are not only used to evaluate trunk stability but are also an exercise used to improve trunk stability. ${ }^{5}$ In recent years, various studies have been reported on posture to maximize the effects of plank exercise performance. ${ }^{6}$ Many studies on effective exercise methods have been reported on not only the general plank position, but also on the use of a sling, Swiss ball, Togu, etc using an unstable support surface, ${ }^{7,8}$ as well as arm angles and changes in various lower extremity conditions etc. ${ }^{910}$

Slings exercises are composed of multifaceted exercises performed in multiple planes and joints through the use of the effects of gravity placed on the body weight, and are difficult to adapt to and maintain balance due to changes in inclination. ${ }^{11}$ Slings are effective and can easily be used to perform exercises to promote muscle strength, neuromuscular control, and stabilization. ${ }^{12}$ 
Elevating lower extremity and hip abduction are used in many ways when exercising on mats. The bridge exercise, similar to plank exercise, promotes muscle activity when Elevating lower extremity and hip abduction. ${ }^{13}$ Likewise, the bridge exercise combined with sling and hip abduction shows higher muscle activity than that of general bridge exercise with sling. ${ }^{14}$

Although there are several recent studies on the effects of changes in lower extremity positions during plank exercises, studies investigating the effects of sling exercises with the inclusion of hip abduction are lacking. Previous studies reported that use of slings during exercises provide an unstable surface and therefore increase muscle activity compared with the general plank exercise,$^{15}$ and plank exercises combined with hip abduction are more effective than the general plank exercises. ${ }^{10}$

Therefore, this study was conducted to investigate the effects of performing plank exercises with hip abduction with use of a sling on trunk muscle activity, to determine which plank exercise position would create the most effective trunk muscle activation, and to propose the steps to be applied to the exercise intervention according to the muscle activities.

\section{METHODS}

\section{Subjects}

This was a cross-sectional study. Twenty-three subjects voluntarily provided their consent after being informed of the study purpose, methods, and application of results. All subjects signed a consent form approved by the Sahmyook university institutional review board.

Muscle activity of healthy subjects were assessed using surface electromyography (EMG) during the maintenance of the knee-supported-in-sling plank, knee-supported-in-sling with one-legged hip abduction plank, ankle-supported-in-sling plank, ankle-supportedin-sling with one-legged hip abduction plank exercises that were

Table 1. General characteristics of the subjects

\begin{tabular}{lc}
\hline Characteristic & Subjects \\
\hline Sex (male/female) & $23(14 / 9)$ \\
Age (yr) & $29.3 \pm 5.7^{\mathrm{a}}$ \\
Height $(\mathrm{cm})$ & $170.1 \pm 7.1$ \\
Weight $(\mathrm{kg})$ & $66.4 \pm 12.1$ \\
\hline
\end{tabular}

aMean \pm SD. performed in random order.

The control group was comprised of healthy subjects provided their voluntary informed consent for the experiment. Subjects who experienced lumbago pain within the past 6 months, congenital deformities of the upper and lower extremities, severe orthopedic or neurological disease, or superficial injury or pain were excluded from the study (Table 1).

\section{Experimental methods}

\section{1) Research procedure}

The maximum voluntary isometric contraction was assessed from the multifidus, rectus abdominis, external oblique, and internal oblique muscles from each subject. Prior to the experiment, each subject was provided with a full explanation on how to perform each plank exercise and was allowed to practice each plank exercise at least 3 times to become familiar with the exercise methods. Each of the plank exercises was listed below and was performed three times each in random order.

\section{(1) General plank position}

For the starting position, the subjects had the upper arms spread shoulder-width apart, and the upper arms parallel to the elbows. According to the instructions of the researcher, subjects were asked to maintain maximal knee extension with the toes pointed in plantar flexion. Subjects were to have the head, trunk, and leg in alignment (Figure 1A). ${ }^{4}$

\section{(2) One-legged hip abduction plank position}

For the starting position, the subjects had the upper arms spread shoulder-width apart, and the upper arms parallel to the elbows. The subjects were instructed to maintain maximal knee extension with the toes pointed in plantar flexion. Subjects were to have the head, trunk, and lower extremity in alignment. The right lower extremity was elevated above the level of the buttocks and placed at 30 degrees of hip abduction (Figure 1B).

\section{(3) Knee supported-in-sling plank position}

From the general plank position, the subjects had the upper arms spread shoulder-width apart and the upper arms parallel to the elbows. Subjects were instructed to maintain maximal knee extension with the head, trunk, and lower extremity in alignment (Figure 2A). 

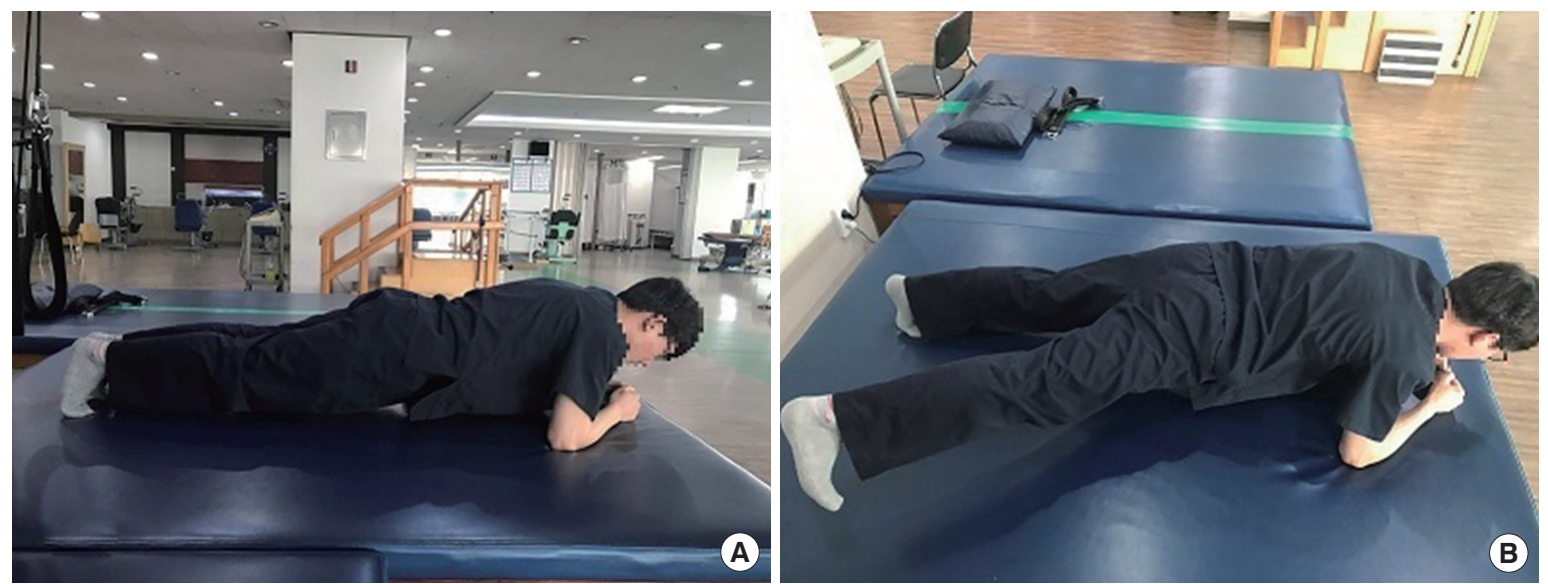

Figure 1. General plank position (A) \& one-legged hip abduction plank position (B).
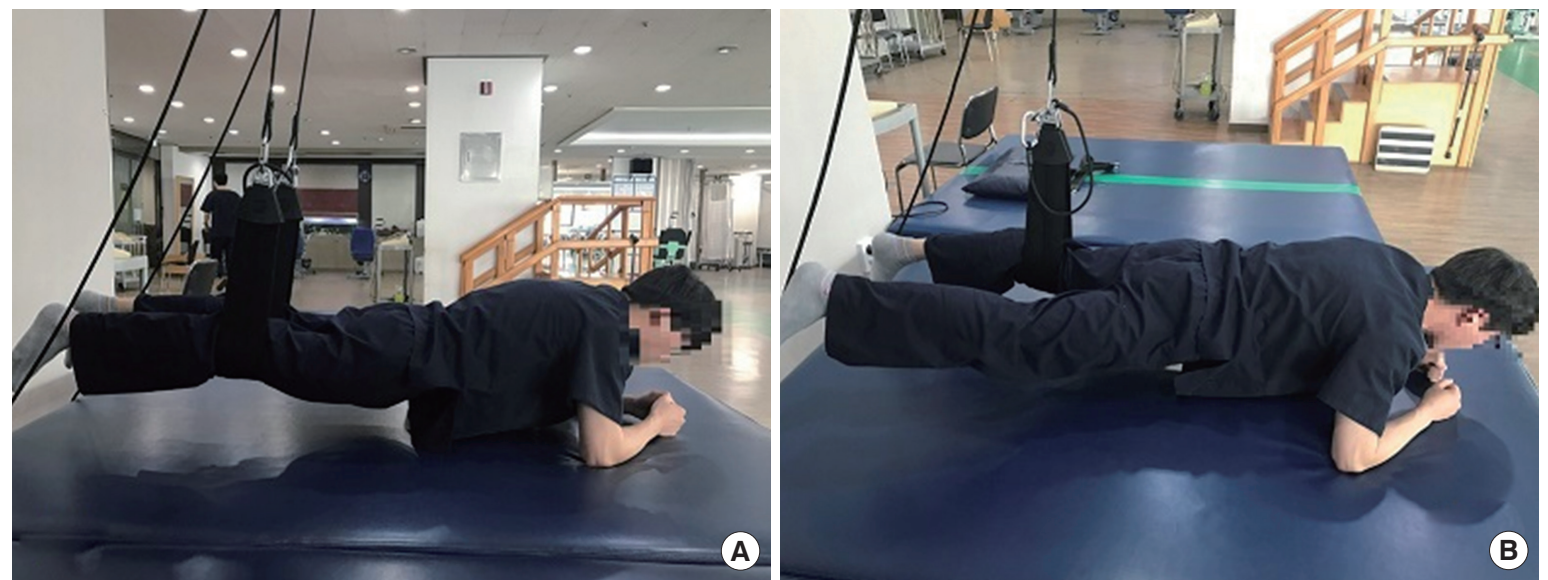

Figure 2. Knee supported-in-sling plank position (A) \& knee supported-in-sling with one-legged hip abduction plank position (B).
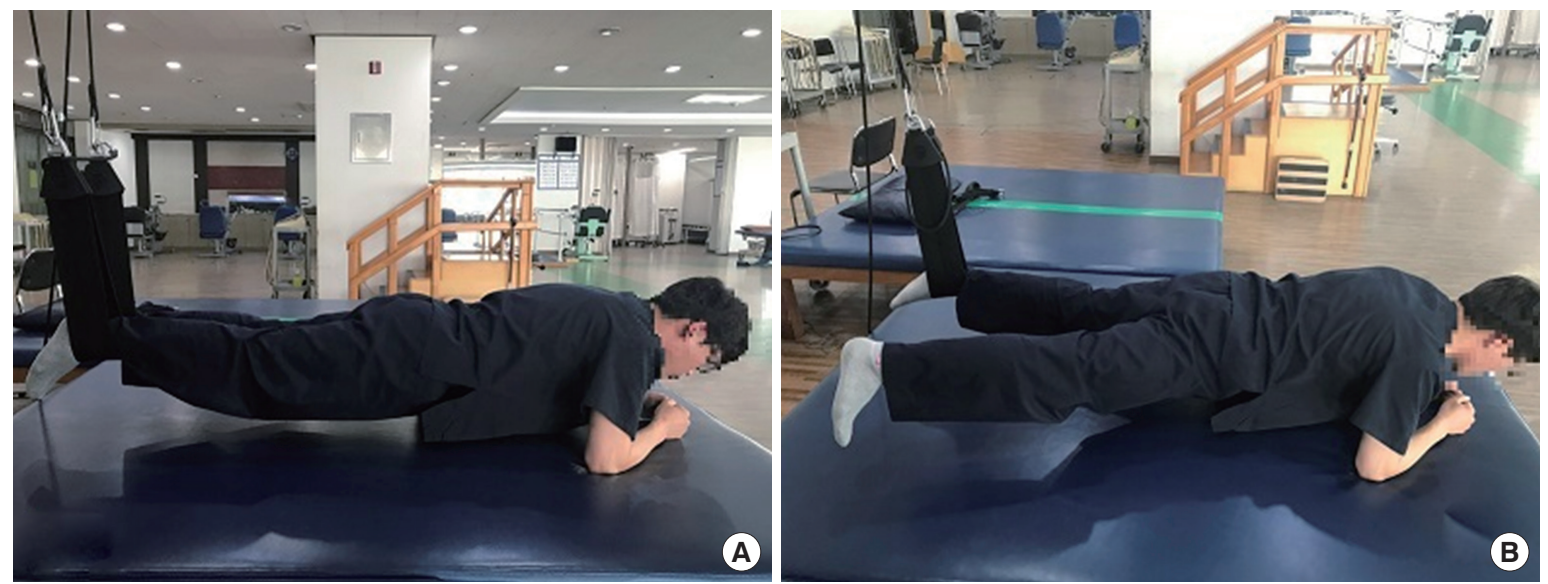

Figure 3. Ankle supported-in-sling plank position (A) \& ankle supported-in-sling with one-legged hip abduction plank position (B).

(4) Knee supported-in-sling with one-legged hip abduction plank position

From the general plank position, the subject's right knee was placed in a sling at shoulder height, their upper arms spread shoulder- width apart, and the upper arms were parallel to the elbows. Subjects were instructed to perform maximal left knee extension with their head, trunk, and left lower extremity in alignment. The right lower extremity was placed in 30 degrees hip abduction while raised 
to the level of the left lower extremity (Figure 2, 2B).

\section{(5) Ankle supported-in-sling plank position}

From the general plank position, bilateral ankles were placed in a sling at shoulder height, upper arms spread shoulder-width apart, and the upper arms were parallel to the elbows. Subjects were instructed to perform maximal knee extension with their head, trunk, and lower extremity in alignment (Figure 3A).

(6) Ankle supported-in-sling with one-legged hip abduction plank position From the general plank position, the left ankle was placed in a sling at shoulder height, upper arms spread shoulderwidth apart, and the upper arms were parallel to the elbows. Subjects were instructed to perform maximal knee extension with their head, trunk, and lower extremity in alignment. The right lower extremity was placed in 30 degrees of hip abduction while raised to the level of the left lower extremity (Figure 3B).

While waiting in prone on elbow position, upon cue the subjects assumed into the position with the head, trunk, and lower extremity in alignment. Each condition was repeated 3 times with a 1-minute rest period in between and measurements were performed in random order.

\section{2) Measurement}

\section{(1) Collection of EMG materials}

The Telemyo 2400 G2 Telemetry EMG system (Noraxon, USA, 2011) was used in order to examine the muscular activity of subjects. The sampling rate was $100 \mathrm{~Hz}$ and the bandwidth filter was set at $10-450 \mathrm{~Hz}$.

For the multifidus, two active electrodes are placed parallel to the spine, $2 \mathrm{~cm}$ apart, approximately $2 \mathrm{~cm}$ from the spine over the muscle mass. The iliac crest may be used to locate the L-3 vertebra. For the rectus abdominis, the electrodes are placed $3 \mathrm{~cm}$ apart and parallel to the muscle fibers of rectus so that they are located approximately $2 \mathrm{~cm}$ lateral and across from the umbilicus over the muscle belly. For the external oblique, the electrodes were placed $15 \mathrm{~cm}$ lateral to the umbilicus. For the internal oblique, the electrodes were placed $2 \mathrm{~cm}$ below and $2 \mathrm{~cm}$ medial to the anterior superior iliac crest. $^{16}$

Prior to the experiment, muscle contraction of the multifidus, rectus abdominis, external and internal oblique were assessed for 5 seconds and analyzed in order to obtain the MVIC.

For the multifidus, subjects were asked to lift their trunk up from a prone position with the lower extremities fixated by the therapist. For the external and internal oblique, subjects were asked to lift their trunk up and diagonally from a supine position while their lower extremities were fixated. For the rectus abdominis, the subjects were asked to raise their trunk up from a supine position and with their lower extremities fixated. ${ }^{16}$

\section{(2) Data and statistical analysis}

The muscle activity signals were processed by the Myoresearch XP Master edition version 1.08.12 (Noraxon Inc, Arizona, USA, 2011), and after undergoing full-wave rectification, the RMS (root mean square) values were taken and divided by the MVIC values and the \%MVIC was used. With the head, trunk, and lower extremity in alignment during the performance of each plank exercise, the muscle activity was assessed for 5 seconds, and with the exclusion of the first and last second, muscle activity from only the middle three seconds was used for analysis. All plank exercise conditions were repeated three times and average values were normalized to MVIC values.

Descriptive statistics was used to analyze the general characteristics of subjects, and the two-way repeated measures ANOVA was used to investigate the effects of performing various plank exercise conditions on trunk and lower extremity muscle activity.

In order to examine for the differences in trunk and lower extremity muscle activity according to the various plank exercise conditions, a post-hoc analysis with the least significant difference (LSD) was performed. Significance level was set at $\mathrm{p}<0.05$.

\section{RESULTS}

1. The differences in muscle activity according to the various plank exercise positions and methods were as follows. There was no significant interaction or impact on muscle activity of all muscles with the inclusion of the one-legged hip abduction method. There was a significant difference in muscle activity of the multifidus and internal oblique on the supported lower extremity side, and the multifidus, external, and internal oblique of the elevated lower extremity side $(\mathrm{p}<0.05)$. There was a significant difference in the muscle activities of the multifidus, rectus abdominis, external, and 
Table 2. Comparison of the core muscle activity during plank exercise

\begin{tabular}{|c|c|c|c|c|c|c|c|c|}
\hline & & & \multicolumn{3}{|c|}{ Muscle activation (\%MVIC) } & \multirow{2}{*}{$\begin{array}{c}\text { Main effect } \\
\text { (method) } \\
F(p)\end{array}$} & \multirow{2}{*}{$\begin{array}{c}\begin{array}{c}\text { Main effect } \\
\text { (hip abduction) }\end{array} \\
F(p)\end{array}$} & \multirow{2}{*}{$\begin{array}{c}\begin{array}{c}\text { Interaction } \\
\text { (method* } \\
\text { hip abduction) }\end{array} \\
F(p)\end{array}$} \\
\hline & & & SK & G & SA & & & \\
\hline \multirow[t]{4}{*}{$\mathrm{MF}$} & Ipsilateral & General & $6.85(3.66)$ & $8.75(4.75)^{*}$ & $9.4(5.63)^{*}$ & $4.60(0.012)$ & $38.60(<0.000)$ & $1.13(0.325)$ \\
\hline & & Hip abduction & $15.89(7.68)^{a}$ & $14.9(6.75)^{\mathrm{a}}$ & $17.67(7.96)^{\mathrm{a}, t}$ & & & \\
\hline & Contralateral & General & $6.85(3.66)$ & $7.8(5.48)$ & $6.96(3.9)$ & $0.25(0.779)$ & $25.81(<0.000)$ & $0.21(0.813)$ \\
\hline & & Hip abduction & $12.88(9.72)^{\mathrm{a}}$ & $12.97(8.24)^{\mathrm{a}}$ & $12.92(8.27)^{\mathrm{a}}$ & & & \\
\hline \multirow[t]{4}{*}{ RA } & Ipsilateral & General & $28.58(17.39)$ & $43.31(20.99)^{*}$ & $54.49(26.67)^{*,+}$ & $25.96(<0.000)$ & $0.68(0.411)$ & $0.92(0.399)$ \\
\hline & & Hip abduction & $25.69(15.76)$ & $46.01(22.02)^{\star}$ & $63.67(23.08)^{* .+}$ & & & \\
\hline & Contralateral & General & $26.29(15.43)$ & $42.1(20.27)^{\star}$ & $53.54(25.46)^{*,+}$ & $27.22(<0.000)$ & $0.17(0.678)$ & $0.50(0.610)$ \\
\hline & & Hip abduction & $22.58(15.92)$ & $42.48(19.79)^{*}$ & $59.66(23.18)^{*,+}$ & & & \\
\hline \multirow[t]{4}{*}{ EO } & Ipsilateral & General & $16.34(8.87)$ & $26.06(9.48)^{*}$ & $29.44(13.53)^{*,+}$ & $14.69(<0.000)$ & $58.17(<0.000)$ & $1.14(0.324)$ \\
\hline & & Hip abduction & $33.65(17.28)^{\mathrm{a}}$ & $40.73(16.62)^{a, *}$ & $53.82(19.37)^{a, *,+}$ & & & \\
\hline & Contralateral & General & $16.55(11.2)$ & $24.95(10.01)^{*}$ & $28.73(14.04)^{*,+}$ & $8.3(<0.000)$ & $3.12(0.08)$ & $0.09(0.915)$ \\
\hline & & Hip abduction & $19.8(18.94)$ & $30.83(16.28)^{*}$ & $34.21(22.95)^{* .+}$ & & & \\
\hline \multirow[t]{4}{*}{10} & Ipsilateral & General & $32.54(16.15)$ & $43.99(19.24)^{*}$ & $54.92(22.01)^{*,+}$ & $16.29(<0.000)$ & $8.05(0.005)$ & $0.11(0.898)$ \\
\hline & & Hip abduction & $41.9(20.06)^{\mathrm{a}}$ & $51.71(20.84)^{a}$ & $66.46(19.7)^{a, *} *+$ & & & \\
\hline & Contralateral & General & $28.28(14.41)$ & $42.26(20.02)^{*}$ & $50.09(25.25)^{*,+}$ & $9.94(<0.000)$ & $9.55(0.002)$ & $0.13(0.882)$ \\
\hline & & Hip abduction & $42.29(28.11)^{\mathrm{a}}$ & $51.6(23.78)^{a}$ & $62.35(21.28)^{a, *, t}$ & & & \\
\hline
\end{tabular}

Value are presented as mean (SD).

SK: knee supported-in-sling plank position, G: general plank position, SA: ankle supported-in-sling plank position, MF: multifidus, RA: rectus abdominis, EO: external oblique, IO: internal oblique.

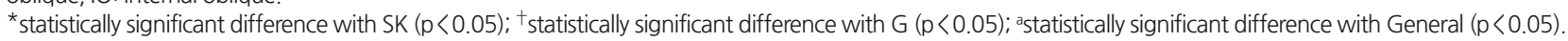

internal oblique of the supported side and the rectus abdominis, external, and internal oblique of the elevated side $(\mathrm{p}<0.05)$ (Table 2).

2. Post-hoc analysis results (comparison of the three exercises with lower extremity elevation, non-elevation) (Table 2).

1) Muscle activities of the rectus abdominis and external oblique on the elevated side and the rectus abdominis, external, and internal oblique of the supported side were significant in the one-legged hip abduction with the ankle-supported-in-sling plank compared with the general plank method $(\mathrm{p}<0.05)$.

Muscle activity of the multifidus on the elevated side was significant with the ankle-supported-in-sling compared with the general plank position $(\mathrm{p}<0.05)$. In addition, muscle activities of the rectus abdominis, external oblique, and internal oblique of the supported side and the rectus abdominis and external oblique of the elevated side were significant with the general plank compared with the knee-supported-in-sling plank method $(\mathrm{p}<0.05)$.

2) Muscle activities of the rectus abdominis and external oblique of the elevated side, and rectus abdominis, external, and internal oblique of the supported side were significant with the bilateral an- kles-supported-in-sling plank exercises compared with the general and knee-supported-in-sling exercises $(\mathrm{p}<0.05)$. There was a significant increase in muscle activity of the support side and the internal oblique of the elevated side in the ankle-supported-in-sling plank compared with the knee-supported-in-sling method $(\mathrm{p}<0.05)$. In addition, muscle activities of the rectus abdominis, external, and internal oblique of the supported side and the multifidus, rectus abdominis, internal and external oblique were significant with the general plank compared to the knee-supported-in-sling plank $\operatorname{method}(\mathrm{p}<0.05)$.

\section{DISCUSSION}

This study investigated the changes in muscle activity of the core muscles, multifidus, rectus abdominis, external, and internal oblique, during the performance of plank exercises with the addition of a sling and hip abduction.

Results have shown that there was a significant difference in muscle activities of the multifidus, external, and internal oblique of the elevated side when performed with one lower extremity supported compared with two lower extremities supported, and de- 
pending on the plank exercise methods, muscle activities of the multifidus, rectus abdominis, external, and internal oblique of the supported side and the rectus abdominis, external, and internal oblique of the elevated side were significant. In addition, post-hoc analysis of the various plank exercise methods showed that muscle activity was significantly increased with the general plank compared to the knee-supported-in-sling plank position, and with the ankle-supported-in-sling plank compared with the general plank exercise method. These results are compatible with the results from previous studies.

A study by Lee compared the general plank versus a plank exercise performed on an unstable surface and found that muscle activities of the left external oblique muscle, right rectus abdominal muscle, and the left rectus abdominal muscle were higher with the ankle-supported-in-sling plank compared to the general plank exercise methods. Atsushi8 also found that the trunk muscle activities were significantly higher during the performance of a plank exercise method on an unstable surface with use of a Swiss ball compared with the general plank exercise method.

There shows higher muscle activity in plank with sling than the muscle activity in general plank in this study as well. Both side (supporting and elevation) rectus abdominis, external oblique, internal oblique, show higher muscle activity.

Mok et al. ${ }^{17}$ stated that slings provide an unstable surface and therefore, is more effective for trunk muscle training, and that activities performed on an unstable surface produce increased core muscle activity and stabilization compared with use of a stable surface. ${ }^{18}$

When reviewing the literature on the comparison of muscle activities during the performance of the general plank and the onelegged hip abduction plank positions, Bak ${ }^{19}$ stated that muscle activity was significantly higher during bride exercises performed with one-legged hip abduction compared with the general bridge exercise. $\mathrm{Choi}^{20}$ also found that muscle activity was significantly higher during the performance of a bridge with one-legged hip abduction and with one-legged hip abduction and use of a sling compared with the general bridge and general bridge with use of a sling exercise. The increases in muscle activity may have been observed due to compensation for instability caused by the decreased bodily contact with the ground. ${ }^{21}$

Significant increases in muscle activity during the general plank exercise method compared with the knee-supported-in sling plank is considered to be due to the role of leverage. One of the functions of levers are that it converts power into torque, and that greater the center-to-center distance, the greater the torque. ${ }^{22}$ Therefore, the decrease in power that occurred during the knee-supported-in-sling position is considered to be due to the decreased center-to-center distance and thus, a shorter lever arm, compared with the general plank and ankle-supported-in-sling plank positions. Similarly, it is considered that the increases in power observed during the onelegged hip abduction plank compared with the bilaterally supported lower-extremity plank positions were due to the increases in lever arm length.

This study showed that the muscle activity of the rectus abdominis during the one-legged hip abduction plank exercises were not significant. This is believed to be due to the anatomical differences of the rectus abdominis. ${ }^{22}$ According to Neumann, ${ }^{22}$ the rectus abdominis is arranged widely in a vertical plane while the external and internal oblique are anatomically arranged in an outer or posterolateral direction toward midline within the trunk, and therefore, the structure of the rectus abdominis is difference.

The limitations of this study are as follows. Firstly, the results of this study cannot be generalized due to the small number of healthy subjects. Secondly, the learning effect could not be ruled out due to the subjects having to perform 6 various activities at one point of time. Through this study, it has been found that plank exercises can be performed with different range of intensities and that the level of difficulty can be selected according to trunk muscle strength. Those who just begin exercise or lack of muscle strength should apply sling on the knee, while professional athletes or those who need powerful core exercise should apply sling on the ankle to have higher muscle activity.

Further studies should consider evaluating changes in trunk muscle strength, muscle thickness, and fatigue, and be conducted as an experimental study versus a cross-sectional study.

\section{REFERENCES}

1. Kisner C, Colby LA. Therapeutic exercise : foundations and techniques. 6th ed. Philadelphia, F.A. Davis, 2012: 409-538.

2. Kim EJ, Hwang BY, Kim JH. Effect of core strength exercises on balance and walking in patients with stroke. J Kor Soc Phys Ther. 2009;21(4):1722.

3. Queiroz BC, Cagliari MF, Amorim CF et al. Muscle activation during four pilates core stability exercises in quadruped position. Arch Phys 
Med Rehabil. 2010;91(1):86-92.

4. Schoenfeld BJ, Contreras B, Tiryaki-Sonmez G et al. An electromyographic comparison of a modified version of the plank with a long lever and posterior tilt versus the traditional plank exercise. Sports Biomech. 2014;13(3):296-306.

5. Hodges PW, Richardson CA. Feedforward contraction of transversus abdominis is not influenced by the direction of arm movement. Exp Brain Res. 1997;114(2):362-70.

6. Kang KW, Son SM, Ko YM. Changes in abdominal muscle thickness and balance ability on plank exercises with various surfaces. J Kor Phys Ther. 2016:28(5):264-8.

7. Lee J, Jeong KH, Lee HA et al. Comparison of three different surface plank exercises on core muscle activity. Phys Ther Rehabil Sci. 2016;5 (1):29-33.

8. Imai A, Kaneoka K, Okubo Y et al. Trunk muscle activity during lumbar stabilization exercises on both a stable and unstable surface. J Orthop Sports Phys Ther. 2010;40(6):369-75.

9. Schoenfeld BJ, Contreras B, Tiryaki-Sonmez G et al. An electromyographic comparison of a modified version of the plank with a long lever and posterior tilt versus the traditional plank exercise. Sports Biomech. 2014;13(3):296-306

10. Kang MH, Kim SY, Kang MJ et al. Effects of isometric hip movements on electromyographic activities of the trunk muscles during plank exercises. J Phys Ther Sci. 2016;28(8):2373-5.

11. Shin YA. Comparison of core stabilizer muscle activity according to movement difficulty and stability during various TRX plank. J Kinesiol. 2016;16(4):31-41.

12. Kim KS, Shin HK. The Effects of the sling strap height on trunk and hip muscle activation during the bridging exercise with sling. J Kor Phys
Ther. 2016:28(1):59-63.

13. Cho MK, Bak JW, Chung YJ. The effects of performing a one-legged bridge with use of a sling on trunk and gluteal muscle activation. Phys Ther Rehabil Sci. 2016;5(2):70-7.

14. Park HJ, Oh DW, Kim SY. Effects of integrating hip movements into bridge exercises on electromyographic activities of selected trunk muscles in healthy individuals. Man Ther. 2014;19(3):246-51.

15. Snarr RL, Esco MR. Electromyographical comparison of plank variations performed with and without instability devices. J Strength Cond Res. 2014;28(11):3298-305.

16. Cram JR, Kasman GS, Holtz J. Introduction to surface electromyography. Gaithersburg, Md., Aspen Publishers, 1998:245-381.

17. Mok NW, Yeung EW, Cho JC et al. Core muscle activity during suspension exercises. J Sci Med Sport. 2015;18(2):189-94.

18. Vera-Garcia FJ, Elvira JL, Brown SH et al. Effects of abdominal stabilization maneuvers on the control of spine motion and stability against sudden trunk perturbations. J Electromyogr Kinesiol. 2007;17(5):556-67.

19. Bak JW, Cho MK, Chung YJ. The effects of performing a one-legged bridge with hip abduction and unstable surface on trunk and gluteal muscle activation in healthy adults. J Kor Phys Ther. 2016:28(3):205-11.

20. Choi KJ, Bak JW, Cho M et al. The effects of performing a one-legged bridge with hip abduction and use of a sling on trunk and lower extremity muscle activation in healthy adults. J Phys Ther Sci. 2016;28(9):26258 .

21. Shumway-Cook A, Woollacott MH. Motor control: translating research into clinical practice. 4th ed. Philadelphia, Wolters Kluwer Health/Lippincott Williams \& Wilkins, 2012:157-258.

22. Neumann DA. Kinesiology of the musculoskeletal system: foundations for physical rehabilitation. St. Louis, Mosby, 2002:311-51. 